\title{
Controlled Grain Growth Applied to the Problem of Grain Boundary Energy Measurements
}

\author{
C. G. DUNN,* Member AIME
}

ThE measurement of interfacial tensions or grain boundary energies in metals is an important metallurgical problem, especially since it bears on growth and nucleation processes. Statistical methods of measuring equilibrium grain boundary angles, which relate interfacial tensions, have been used by some investigators ${ }^{1,2}$ but more direct methods would be preferred.

A direct way, based on a simple extension of a successful method of growing single crystals of silicon ferrite to predetermined orientations in flat specimens, ${ }^{3}$ is to produce three-grain specimens in such a way that the equilibrium common grain boundary will be perpendicular to the surface of the specimen. The angles to be measured then appear as the grain boundary angles in the surface of the specimen.

The method of producing a single crystal specimen with a predetermined orientation is basically the same as that employed by Yoshida and Fujiwara $^{4}$ in their work on aluminum wires. The specimen, prior to any single crystal transformation, must have a matrix which will support the growth of a crystal of the desired orientation. Growth may occur through exaggerated grain growth or, if the matrix is strained a critical amount, by normal recrystallization. In either case the matrix must have stability at the temperature used to effect the transformation, this stability appearing as a long induction period for exaggerated grain growth or for the formation of recrystallization nuclei. Additional control is gained by using a high temperature gradient through which the specimen is moved at a suitable rate. To obtain the desired orientation, the specimen is fed a short way into the furnace producing one or more crystals in the hot end. After the specimen is removed from the furnace, the orientation of a crystal is determined, so that this crystal may be reoriented by properly deforming a narrow piece of the matrix between the end crystal and the rest of the specimen. This part of the specimen in the case of silicon ferrite is preferably

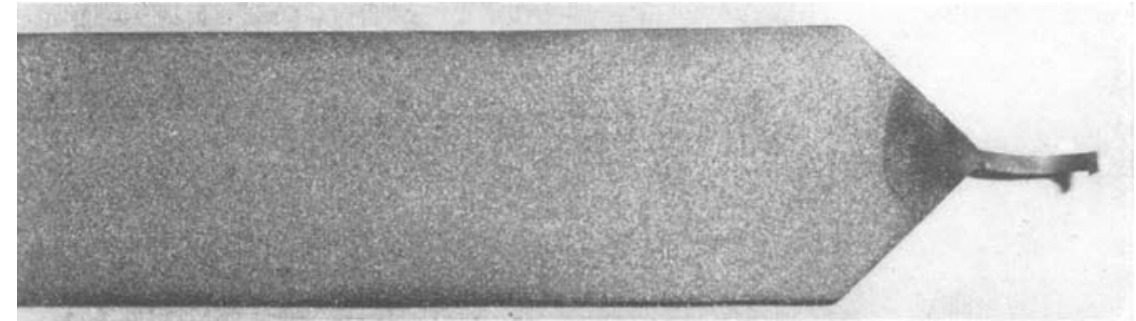

FIG 1-Photograph showing fine grained specimen with reoriented seed crystal in the form of small flag on end of the narrow twisted part of the specimen. Natural size.

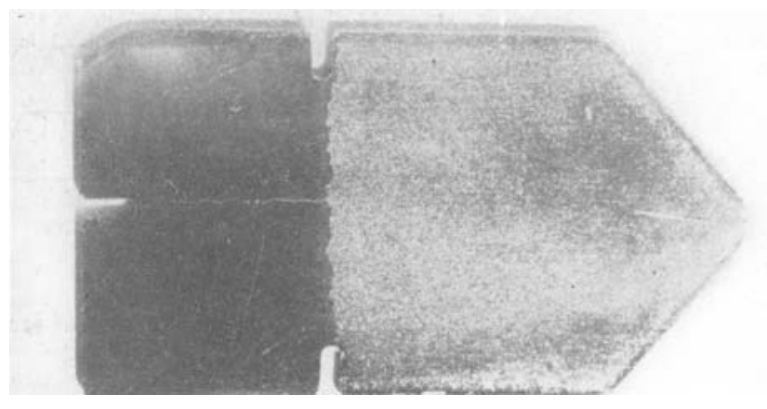

FIG 2-Photograph showing specimen composed of three grains each having a $\{110\}$ plane in the plane of the specimen. Sample ready to be annealed. $\times 2$.

held at red heat during the reorienting process. Fig 1 shows a specimen which is ready to be transformed into a single crystal through growth of a reoriented "seed" crystal.

When the method just described is applied to the production of a threegrain specimen two crystals are grown first side by side in one end, the specimen is then reversed and the third grain grown from the opposite end. A suitable group of grains each of different orientation is one where all three grains have the same crystallographic plane in the plane of the specimen. $\dagger$

Fig 2 shows a photograph of a threegrain group prepared as described. The notches are introduced in the open

† F. Lionetti and the writer are investigating a series of groups all crystals of which have a $\{110\}$ plane in the plane of the specimen. Results of this in
published.

Technical Note No. 9. Manuscript received September 27 , 1948. * Research Physicist, G
tric Co., Pittsfield, Mass.

tric Co., Pittsfield, Mass. paper. ends of the grain boundaries to anchor them during the final annealing operations which ultimately should bring the grain boundaries to the lowest energy configuration.

Two-grain groups with different crystallographic planes in the plane of the specimen can be similarly prepared and treated. If the two grains have different surface (gas to metal) energies per unit area, the equilibrium condition leads to a curved grain boundary.*

* This feature of the problem was pointed out by J. C. Fisher.

\section{References}

1. Cyril Stanley Smith: Grains, Phases and Interfaces: An Interpretation of Microstructure. Trans. AIME 175, 15. Met. Tech. TP 2387, June 1948.

2. D. Harker and E. Parker: Grain Shape and Grain Growth. Trans. A.S.M. (1945) 34, 156-195.

3. C. G. Dunn, A. Seddon, and R. Wylie:

4. Takeo Fujiwara: A Method of Producing A Long Single-Crystal Wire of Aluminum with any Desired Crystallographic Axis. Jnl. of Sci. Horoshima Univ. A, (1939) 9, No. 3 , 227. Unpublished Work, 1946-1947. 